\title{
Quantum phase transitions and bifurcations: reduced fidelity as a phase transition indicator for quantum lattice many-body systems
}

\author{
Jin-Hua Liu, ${ }^{1}$ Qian-Qian Shi, ${ }^{1}$ Jian-Hui Zhao, ${ }^{1}$ and Huan-Qiang Zhou ${ }^{1}$ \\ ${ }^{I}$ Centre for Modern Physics and Department of Physics, \\ Chongqing University, Chongqing 400044, The People's Republic of China
}

\begin{abstract}
We establish an intriguing connection between quantum phase transitions and bifurcations in the reduced fidelity between two different reduced density matrices for quantum lattice many-body systems with symmetrybreaking orders. Our finding is based on the observation that, in the conventional Landau-Ginzburg-Wilson paradigm, a quantum system undergoing a phase transition is characterized in terms of spontaneous symmetry breaking that is captured by a local order parameter, which in turn results in an essential change of the reduced density matrix in the symmetry-broken phase. Two quantum systems on an infinite lattice in one spatial dimension, i.e., quantum Ising model in a transverse magnetic field and quantum spin 1/2 XYX model in an external magnetic field, are considered in the context of the tensor network algorithm based on the matrix product state representation.

PACS numbers: 03.67.-a, 03.65.Ud, 03.67.Hk
\end{abstract}

Introduction. Recently we have witnessed a growing interest in the study of quantum many-body systems in the context of the fidelity approach to quantum phase transitions (QPTs) [1, 2] with symmetry-breaking/topological orders [3, 4, 5, 6, 7, 8, 9, 10, 11, 12]. In Refs. [4, 5, 6, 7], it has been argued that the ground state fidelity per site may be used to detect QPTs. Since the argument is solely based on the basic Postulate of Quantum Mechanics on quantum measurements, it is expected that this approach is applicable to quantum lattice systems in any spatial dimensions, regardless of what type of internal order is present in quantum manybody states. In fact, it has been confirmed that the ground state fidelity per site is able to describe QPTs arising from an SSB [4, 5, 6, 7, 8], the Kosterlitz-Thouless transition [9] and topological QPTs in the Kitaev model [10]. Remarkably, the ground state fidelity per site may be computed in terms of the newly-developed tensor network (TN) algorithms, such as the matrix product states (MPS) [13, 14, 15] in one spatial dimension, and the tensor product states (TPS) [16], or equivalently, the projected entangled-pair states (PEPS) [17], in two and higher spatial dimensions.

In the conventional Landau-Ginzburg-Wilson paradigm, an SSB, which occurs when a system possesses a certain symmetry whereas the ground state wave functions do not preserve it [18, 19], is quantified in terms of a local order parameter. An intriguing feature of local order parameters for quantum systems with symmetry-breaking orders is bifurcations they exhibit at critical points. Remarkably, such a bifurcation also manifests itself in the ground state fidelity per site [8]. The advantage of the latter over local order parameters lies in the fact that the ground state fidelity per site is universal in the sense that it is not model-dependent, in contrast to model-dependent order parameters in characterizing QPTs in quantum lattice many-body systems.

The investigation that has been carried out so far mainly focuses on the ground state wave functions for quantum lattice systems. Thus the fidelity is equivalent to the overlap between two different ground states, which are pure states.
However, as first discussed in Ref. [6] (see also [12]), the fidelity defined for two different mixed states is also useful to locate phase transition points. The mixed states are described by the reduced density matrices arising from tracing out the degrees of freedom in the environment surrounding a sub-system. Indeed, for a sub-system of a composite quantum system, a reduced density matrix is a basic notion that is indispensable for the analysis of a composite quantum system. In fact, the presence of a local order parameter makes a difference in the reduced density matrices in the symmetric and symmetry-broken phases. From this one may anticipate that the (reduced) fidelity between two different reduced density matrices for quantum lattice many-body systems with symmetry-breaking orders should capture bifurcations arising from an SSB.

In this paper, we attempt to address this problem. We shall investigate two quantum models on an infinite lattice in one spatial dimension, i.e., quantum Ising model in a transverse magnetic field and spin 1/2 XYX model in an external magnetic field. Both systems possess a discrete symmetry group $Z_{2}$, but the ground states break the symmetry. The SSB is reflected as a bifurcation [8] in the ground state reduced fidelity for both systems. Our result demonstrate that one may identify a phase transition point as a bifurcation point in the ground state reduced fidelity between two different reduced density matrices for quantum lattice many-body systems with symmetry-breaking orders [20].

The models. The first model we consider in this paper is the quantum Ising model in a transverse magnetic field in an infinite-size lattice in one spatial dimension. The Hamiltonian takes the form,

$$
H=-\sum_{i=-\infty}^{\infty}\left(S_{x}^{[i]} S_{x}^{[i+1]}+\lambda S_{z}^{[i]}\right),
$$

where $S_{\alpha}^{[i]}(\alpha=x, z)$ are the spin $1 / 2$ Pauli operators at site $i$, and $\lambda$ is the transverse magnetic field. The model is invariant under the symmetry operation: $S_{x}^{[i]} \rightarrow-S_{x}^{[i]}$ and $S_{z}^{[i]} \rightarrow S_{z}^{[i]}$ 
for all sites, which yields the $Z_{2}$ symmetry. As is well known, the system undergoes a second order QPT at the critical field $\lambda_{c}=1[21]$.

The second model is the spin $1 / 2 \mathrm{XYX}$ model in an external magnetic field. The Hamiltonian can be written as

$$
H=\sum_{i=-\infty}^{\infty}\left(S_{x}^{[i]} S_{x}^{[i+1]}+\Delta_{y} S_{y}^{[i]} S_{y}^{[i+1]}+S_{z}^{[i]} S_{z}^{[i+1]}+h S_{z}^{[i]}\right),
$$

where $S_{\alpha}^{[i]}(\alpha=x, y, z)$ are the Pauli spin operators at site $i, \Delta_{y}$ is a parameter describing the anisotropy in the internal space, and $h$ is the external magnetic field. This model also possesses a $Z_{2}$ symmetry, with the symmetry operation: $S_{x}^{[i]} \rightarrow-S_{x}^{[i]}$, $S_{y}^{[i]} \rightarrow-S_{y}^{[i]}$ and $S_{z}^{[i]} \rightarrow S_{z}^{[i]}$ for all sites. Below we shall choose $\Delta_{y}=0.25$. In this case, the critical magnetic field is $h_{c} \sim 3.210(6)$ [22].

The reduced density matrix. We are now in a position to clarify the difference of the reduced density matrices in the symmetric and symmetry-broken phases for both quantum Ising model in a transverse magnetic field and quantum spin $1 / 2$ XYX model in an external magnetic field. The analysis will be carried out for both the one-site and two-site reduced density matrices, respectively. For the quantum Ising model in a transverse magnetic field, the one-site reduced density matrix in the $Z_{2}$ symmetric phase takes the form,

$$
\rho_{\text {ising }}=\frac{1}{2}+2\left\langle S_{z}\right\rangle S_{z},
$$

where $\left\langle S_{z}\right\rangle$ is the expectation value of $S_{z}$ in the ground state in the $Z_{2}$ symmetric phase, whereas the two-site reduced density matrix in the $Z_{2}$ symmetric phase is,

$$
\begin{aligned}
\rho_{\text {ising }}= & \frac{1}{4} I+4 \alpha_{1} S_{x} \otimes S_{x}+4 \alpha_{2} S_{z} \otimes S_{z} \\
& +\alpha_{3} I \otimes S_{z}+\alpha_{4} S_{z} \otimes I .
\end{aligned}
$$

Here, $\alpha_{1}=\left\langle S_{x} \otimes S_{x}\right\rangle, \alpha_{2}=\left\langle S_{z} \otimes S_{z}\right\rangle, \alpha_{3}=\left\langle I \otimes S_{z}\right\rangle$, and $\alpha_{4}=\left\langle S_{z} \otimes I\right\rangle$, with $I$ being the identity matrix.

In the $Z_{2}$ symmetry-broken phase, the presence of the nonzero local order parameter $\left\langle S_{x}\right\rangle$ implies that the one-site reduced density matrix takes the form,

$$
\rho_{i s i n g}=\frac{1}{2}+2\left\langle S_{x}\right\rangle S_{x}+2\left\langle S_{z}\right\rangle S_{z} .
$$

Such a violation of the symmetry is also reflected in the twosite reduced density matrix:

$$
\begin{aligned}
\rho_{\text {ising }}= & \frac{1}{4} I+4 \alpha_{1} S_{x} \otimes S_{x}+4 \alpha_{2} S_{z} \otimes S_{z} \\
& +\alpha_{3} I \otimes S_{z}+\alpha_{4} S_{z} \otimes I++4 \alpha_{5} S_{x} \otimes S_{z} \\
& +4 \alpha_{6} S_{z} \otimes S_{x}+\alpha_{7} I \otimes S_{x}+\alpha_{8} S_{x} \otimes I,
\end{aligned}
$$

with $\alpha_{5}=\left\langle S_{x} \otimes S_{z}\right\rangle, \alpha_{6}=\left\langle S_{z} \otimes S_{x}\right\rangle, \alpha_{7}=\left\langle I \otimes S_{x}\right\rangle$, and $\alpha_{8}=\left\langle S_{x} \otimes I\right\rangle$.

For the quantum spin 1/2 XYX model in an external magnetic field, the one-site reduced density matrix in the $Z_{2}$ symmetric phase takes the form,

$$
\rho_{X Y X}=\frac{1}{2}+2\left\langle S_{z}\right\rangle S_{z},
$$

while the two-site reduced density matrix is

$$
\begin{aligned}
\rho_{X Y X}= & \frac{1}{4} I+4 \beta_{1} S_{x} \otimes S_{x}+4 \beta_{2} S_{y} \otimes S_{y} \\
& +4 \beta_{3} S_{z} \otimes S_{z}+\beta_{4} I \otimes S_{z}+\beta_{5} S_{z} \otimes I,
\end{aligned}
$$

with $\beta_{1}=\left\langle S_{x} \otimes S_{x}\right\rangle, \beta_{2}=\left\langle S_{y} \otimes S_{y}\right\rangle, \beta_{3}=\left\langle S_{z} \otimes S_{z}\right\rangle, \beta_{4}=$ $\left\langle I \otimes S_{z}\right\rangle$, and $\beta_{5}=\left\langle S_{z} \otimes I\right\rangle$.

In the symmetry-broken phase, the one-site reduced density matrix becomes

$$
\rho_{X Y X}=\frac{1}{2}+2\left\langle S_{x}\right\rangle S_{x}+2\left\langle S_{z}\right\rangle S_{z},
$$

whereas the two-site reduced density matrix is

$$
\begin{aligned}
\rho_{X Y X}= & \frac{1}{4} I+4 \beta_{1} S_{x} \otimes S_{x}+4 \beta_{2} S_{y} \otimes S_{y}+4 \beta_{3} S_{z} \otimes S_{z} \\
& +\beta_{4} I \otimes S_{z}+\beta_{5} S_{z} \otimes I+4 \beta_{6} S_{x} \otimes S_{z} \\
& +4 \beta_{7} S_{z} \otimes S_{x}+\beta_{8} I \otimes S_{x}+\beta_{9} S_{x} \otimes I,
\end{aligned}
$$

with $\beta_{6}=\left\langle S_{x} \otimes S_{z}\right\rangle, \beta_{7}=\left\langle S_{z} \otimes S_{x}\right\rangle, \beta_{8}=\left\langle I \otimes S_{x}\right\rangle$, and $\beta_{9}=\left\langle S_{x} \otimes I\right\rangle$.

Bifurcations in the reduced fidelity between two reduced density matrices and quantum phase transitions. The reduced fidelity measures the distance between two quantum mixed states. Specifically, for two reduced density matrices $\rho_{\lambda}$ and $\rho_{\lambda^{\prime}}$, the reduced fidelity $F\left(\rho_{\lambda}, \rho_{\lambda^{\prime}}\right)$ is defined to be

$$
F\left(\rho_{\lambda}, \rho_{\lambda^{\prime}}\right)=\operatorname{tr} \sqrt{\rho_{\lambda}^{1 / 2} \rho_{\lambda^{\prime}} \rho_{\lambda}^{1 / 2}} .
$$

Here, $\rho_{\lambda}$ and $\rho_{\lambda^{\prime}}$ are the reduced density matrices corresponding to two different values, $\lambda$ and $\lambda^{\prime}$, of the control parameter $\lambda$. Notice that the reduced fidelity $F\left(\rho_{\lambda}, \rho_{\lambda^{\prime}}\right)$ is a function of $\lambda$ and $\lambda^{\prime}$, which satisfies the following properties: (i) normalization $F\left(\rho_{\lambda}, \rho_{\lambda}\right)=1$; (ii) symmetry $F\left(\rho_{\lambda}, \rho_{\lambda^{\prime}}\right)=F\left(\rho_{\lambda^{\prime}}, \rho_{\lambda}\right)$; (iii) range $0 \leq F\left(\rho_{\lambda}, \rho_{\lambda^{\prime}}\right) \leq 1$.

The connection between a bifurcation point in the ground state partial fidelity between two reduced density matrices and a critical point for quantum lattice systems undergoing QTPs with symmetry-breaking order may be established in the following way. In the conventional Landau-Ginzburg-Wilson paradigm, a quantum system undergoing a QPT is characterized in terms of an SSB that is captured by a local order parameter, which in turn results in an essential change of the reduced density matrix in the symmetry broken phase, as seen above. More precisely, consider a quantum system on an infinite lattice in one spacial dimension, with $Z_{2}$ as a symmetry group [23]. Suppose the $Z_{2}$ symmetry is spontaneously broken when the control parameter $\lambda$ crosses a critical point $\lambda=\lambda_{c}$, then the system undergoes a QPT with a nonzero local order parameter in the symmetry-broken phase. Such a local order parameter is defined in a local area $\Omega$ on a lattice, and it is not invariant under the action of the symmetry operation generating the symmetry group $Z_{2}$, thus yielding degenerate ground states in the symmetry-broken phase. Therefore, the reduced fidelity $F\left(\rho_{\lambda}, \rho_{\lambda^{\prime}}\right)$, with the reference state $\rho_{\lambda^{\prime}}$ in the symmetry-broken phase, yields two different values, which 
correspond to two distinct values of the local order parameter arising from two degenerate ground states, if $\rho_{\lambda}$ is in the symmetry-broken phase, and one value, if $\rho_{\lambda}$ is in the symmetric phase. That is, a bifurcation point occurs in the ground state reduced fidelity between two reduced density matrices $F\left(\rho_{\lambda}, \rho_{\lambda^{\prime}}\right)$, with the critical point being the bifurcation point. However, if we choose the reference state $\rho_{\lambda^{\prime}}$ in the symmetric phase, then no such a bifurcation occurs, due to the invariance of the reduced density matrix $\rho_{\lambda^{\prime}}$ under the action of the symmetry operation. In addition, the same argument applies to the reduced fidelity defined on any local area $\Omega^{\prime}$ with $\Omega$ as a subset [24].

The results. In the context of the $\mathrm{TN}$ algorithm initiated by Vidal [25], the problem to find the system's ground state wave functions amounts to computing the imaginary time evolution for a given initial state $|\Psi(0)\rangle$ : $|\Psi(\tau)\rangle=$ $\exp (-H \tau)|\Psi(0)\rangle /|\exp (-H \tau)| \Psi(0)\rangle \mid$. An efficient way to achieve this task is to exploit the Suzuki-Trotter decomposition [26], which allows us to reduce the imaginary time evolution operation to a product of two-site evolution operators acting on sites $i$ and $i+1: U(i, i+1)=\exp \left(-h^{[i, i+1)]} \delta \tau\right)$, $\delta \tau<<1$. In addition, any wave function admits an MPS representation in a canonical form: attached to each site is a three-index tensor $\Gamma_{A l r}^{S}$ or $\Gamma_{B l r}^{S}$, and to each bond a diagonal singular value matrix $\lambda_{A}$ or $\lambda_{B}$, depending on the evenness and oddness of the $i$-th site and the $i$-th bond. Here, $s$ is a physical index, $s=1, \cdots, d$, with $d$ being the dimension of the local Hilbert space, and $l$ and $r$ denote the bond indices, $l, r=1, \cdots, \chi$, with $\chi$ being the truncation dimension. The action of a two-site gate $U(i, i+1)$ may be absorbed by performing a singular value decomposition, thus resulting in the update of the MPS representation. Repeating this procedure until the ground state energy converges, one may generate the ground state wave functions in the MPS representation. We emphasize that, in practice, we adjust the truncation dimension $\chi$ to identify a critical point from bifurcation points in the reduced fidelity $F\left(\rho_{\lambda}, \rho_{\lambda^{\prime}}\right)$. In fact, it saves a lot of the computational resources to perform simulations for relatively small values of $\chi$. Usually, a shift in the bifurcation points occurs due to the finiteness of $\chi$. Therefore, it is necessary to perform an extrapolation with respect to $\chi$ to locate the critical point.

In Fig1(a), we plot the ground state reduced fidelity $F\left(\rho_{\lambda}, \rho_{\lambda^{\prime}}\right)$ between the one-site reduced density matrices for the quantum Ising model in a transverse field with the field strength $\lambda$ as the control parameter. Here, we choose $\rho_{\lambda^{\prime}}\left(\lambda^{\prime}=\right.$ $0.9)$ as a reference state, which breaks the $Z_{2}$ symmetry. The one-site reduced fidelity can distinguish two degenerate ground states with a bifurcation point as a pseudo phase transition point $\lambda_{\chi}$ [8]. When the parameter $\lambda$ is tuned beyond such a pseudo transition point, two degenerate ground states vanish, implying that the system undergoes a phase transition. One observes that, with $\chi$ increasing, the pseudo phase transition point $\lambda_{\chi}$ moves toward the exact value 1 . Performing an extrapolation of $\lambda_{\chi}$ with respect to $\chi$, we get $\lambda_{c}=1.00233$. In Fig 1 (b), we show the two-site reduced fidelity for the quantum Ising model in a transverse magnetic field. The same ref-

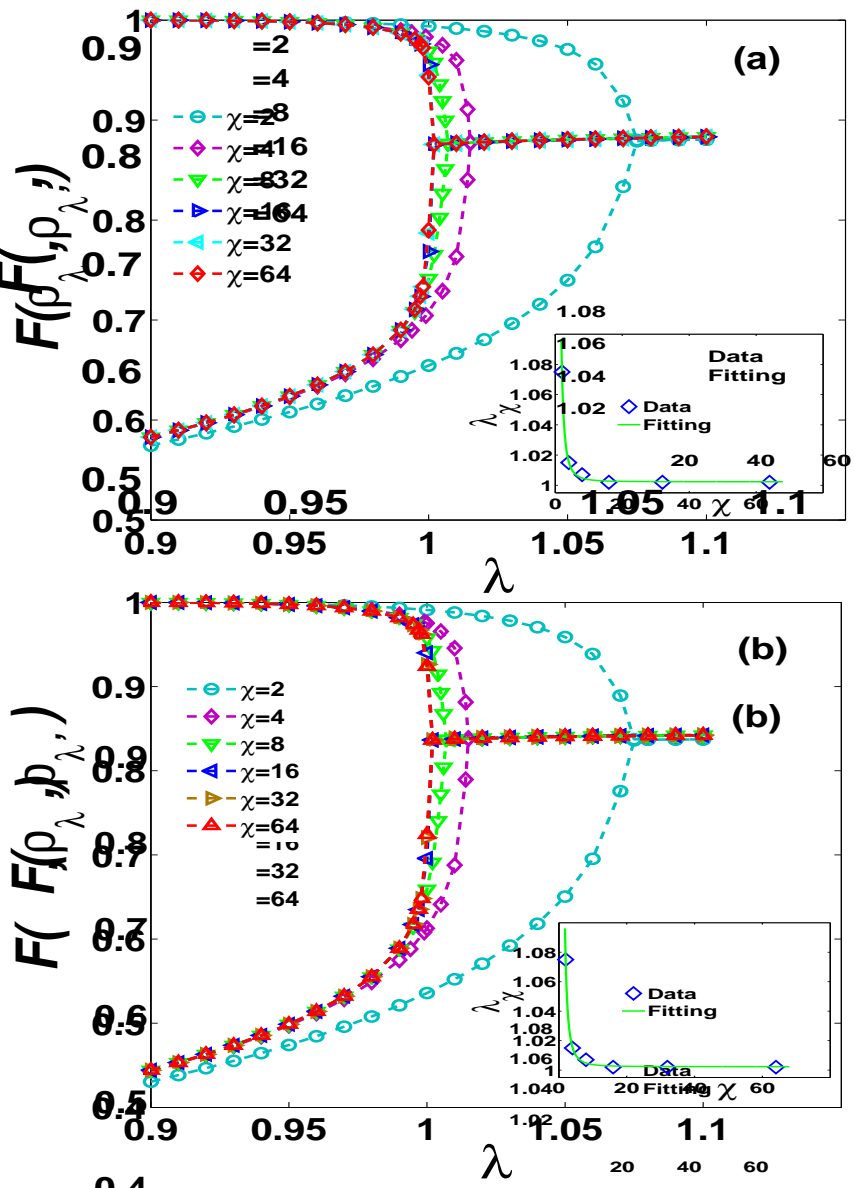

FIG. 1: (color online) (a) The ground state one-site reduced fidelity $F\left(\rho_{\lambda}, \rho_{\lambda^{\prime}}\right)$ for the quantum Ising model in a transverse magnetic field. We choose $\rho_{\lambda^{\prime}}$ (with $\lambda^{\prime}=0.9$ ) as the reference state, which is in the $Z_{2}$ symmetry-broken phase. The pseudo phase transition point $\lambda_{\chi}$ occurs as a bifurcation point. When we enlarge the truncation dimension $\chi$, the pseudo phase transition point $\lambda_{\chi}$ is getting closer to the exact value. Inset: the critical point $\lambda_{c}$ is determined from an extrapolation of the pseudo phase transition point $\lambda_{\chi}$ with respect to the truncation dimension $\chi$. Here, the fitting function is $\lambda_{\chi}=$ $\lambda_{c}+a \chi^{-b}$, with $\lambda_{c}=1.00233, a=0.39373$ and $b=2.43904$. (b) The ground state two-site reduced fidelity, $F\left(\rho_{\lambda}, \rho_{\lambda^{\prime}}\right)$, for the quantum Ising model in a transverse magnetic field. The same reference state $\rho_{\lambda^{\prime}} \quad\left(\lambda^{\prime}=0.9\right)$ has been chosen as in the case of the one-site reduced fidelity. Both insets indicate the same pseudo critical points for the ground state one-site and two-site reduced density matrices for the system. This is expected due to the fact that they are resulted from the same set of the ground states.

erence state is selected as in the case of the one-site reduced fidelity. We observe that a bifurcation also occurs in the twosite partial fidelity. Indeed, it yields the same pseudo phase transition points $\lambda_{\chi}$, and thus the same critical point $\lambda_{c}$.

In Fig. 2] a), the ground state one-site reduced fidelity $F\left(\rho_{h}, \rho_{h^{\prime}}\right)$ for the quantum spin $1 / 2 \mathrm{XYX}$ model in an external magnetic field $h$ is plotted, with the magnetic field strength $h$ as the control parameter. We choose $\rho_{h^{\prime}}$, with $h^{\prime}=3.05$, in the $Z_{2}$ symmetry-broken phase, as the reference state. The reduced fidelity $F\left(\rho_{h}, \rho_{h^{\prime}}\right)$ distinguishes two degenerate ground 

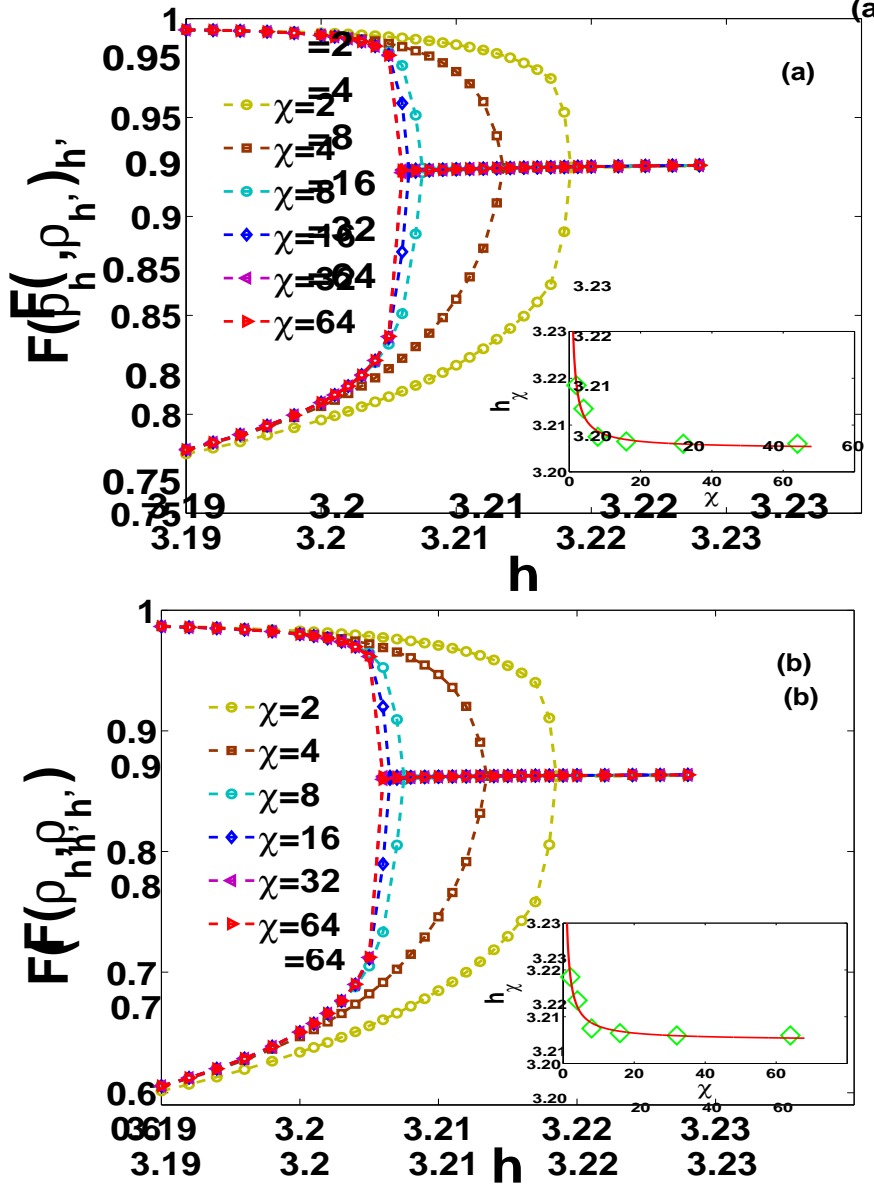

FIG. 2: (color online) (a) The ground state one-site reduced fidelity $F\left(\rho_{h}, \rho_{h^{\prime}}\right)$ for the quantum spin $1 / 2 \mathrm{XYX}$ model in an external magnetic field. Here, the external magnetic field strength $h$ is the control parameter. We choose $\rho_{h^{\prime}}$ ( with $h^{\prime}=3.05$ ) as the reference state, which is in the symmetry-broken phase. The pseudo phase transition point $h_{\chi}$ occurs as a bifurcation point. For a larger value of $\chi$, the pseudo phase transition point $h_{\chi}$ is getting closer to the known value 3.2049. Inset: the critical point $h_{c}$ is determined from an extrapolation of the pseudo phase transition point $h_{\chi}$ with respect to the truncation dimension $\chi$. The fitting function is $h_{\chi}=h_{c}+a \chi^{-b}$, with $h_{c}=3.2049, a=0.0267$ and $b=0.9426$. (b) The ground state twosite reduced fidelity $F\left(\rho_{h}, \rho_{h^{\prime}}\right)$ for the quantum spin $1 / 2$ XYX model. We still choose $\rho_{h^{\prime}}\left(h^{\prime}=3.05\right)$ as the reference state. The two-site reduced fidelity exhibits the similar behavior to that of the one-site reduced fidelity. Both insets display the same pseudo critical points for the one-site and two-site reduced fidelity for the system.

states in the symmetry-broken phase. The bifurcation point $h_{\chi}$ resulted from the one-site reduced fidelity is the pseudo phase transition point, which is quite close to the known critical value $h_{c} \sim 3.210(6)$ [22], if the truncation dimension $\chi$ is large enough. Performing an extrapolation with respect to $\chi$ yields the critical point $h_{c}=3.2049$. In Fig. 2 (b), we plot the two-site reduced fidelity for the quantum XYX model. Here, the same reference state as in the case of the one-site partial fidelity has been chosen. The two-site reduced fidelity $F\left(\rho_{h}, \rho_{h^{\prime}}\right)$ is also able to detect the $Z_{2}$ SSB. Indeed, it again yields the same bifurcation points $h_{\chi}$, and thus the same criti- cal point $h_{c}$.

Summary. We have established an intriguing connection between QPTs and bifurcations in the reduced fidelity between two different reduced density matrices for quantum lattice many-body systems with symmetry-breaking orders. Our work is based on the newly-developed TN algorithms, which produce degenerate ground states in the symmetry-broken phase for quantum lattice systems under QPTs arising from an SSB. Two quantum systems on an infinite lattice in one spatial dimension, i.e., quantum Ising model in a transverse magnetic field and quantum spin 1/2 XYX model in an external magnetic field, have been investigated in the context of the TN algorithm based on the MPS representation.

Acknowledgements. This work is supported in part by the National Natural Science Foundation of China (Grant Nos: 10774197 and 10874252), the Natural Science Foundation of Chongqing (Grant No: CSTC, 2008BC2023).

[1] S. Sachdev, Quantum Phase Transitions, Cambridge University Press, 1999, Cambridge.

[2] X.-G Wen, Quantum Field Theory of Many-Body Systems, Oxford University Press, 2004, Oxford.

[3] P. Zanardi and N. Paunković, Phys. Rev. E 74, 031123 (2006).

[4] H.-Q. Zhou and J.P. Barjaktarević, J. Phys. A: Math. Theor. 41 412001 (2008).

[5] H.-Q. Zhou, J.-H. Zhao, and B. Li, J. Phys. A: Math. Theor. 41 492002 (2008).

[6] H.-Q. Zhou, arXiv:0704.2945

[7] H.-Q. Zhou, R. Orús, and G. Vidal, Phys. Rev. Lett. 100, 080602 (2008).

[8] J.-H. Zhao, H.-L. Wang, B. Li, and H.-Q. Zhou, arXiv:0902.1669

[9] H.-L. Wang, J.-H. Zhao, B. Li, and H.-Q. Zhou, arXiv:0902.1670

[10] J.-H. Zhao, and H.-Q. Zhou, arXiv:0803.0814.

[11] P. Zanardi, M. Cozzini, and P. Giorda, J. Stat. Mech. L02002, (2007); N. Oelkers and J. Links, Phys. Rev. B75, 115119 (2007); M. Cozzini, R. Ionicioiu, and P. Zanardi, Phys. Rev. B76, 104420 (2007); L. Campos Venuti and P. Zanardi, Phys. Rev. Lett. 99, 095701 (2007); W.-L. You, Y.-W. Li, and S.-J. Gu, Phys. Rev. E76, 022101 (2007); S. J. Gu et al., Phys. Rev. B77, 245109 (2008); Y.C. Tzeng and M.F. Yang, Phys. Rev. A77, 012311 (2008); M.F. Yang, Phys. Rev. B76, 180403(R) (2007); J.O. Fjaerestad, J. Stat. Mech. P07011 (2008); S. Chen, L. Wang, Y. Hao, and Y. Wang, Phys. Rev. A77, 032111 (2008).

[12] N. Paunković, P.D. Sacramento, P. Nogueira, V.R. Vieira, and V.K. Dugaev, Phys. Rev. A77, 052302 (2008); H.M. Kwok, C.S. Ho, and S.J. Gu, arXiv:0805.3885, J. Ma, L. Xu, H. Xiong, and X. Wang, arXiv:0805.4062, E. Eriksson and H. Johannesson, arXiv:0902.3848, C. Invernizzi and M. Paris, arXiv:0905.0980, A. Khan and P. Pieri, arXiv:0905.2165.

[13] M. Fannes, B. Nachtergaele, and R.F. Werner, Comm. Math. Phys. 144, 443 (1992); J. Funct. Anal. 120, 511 (1994); S. Östlund and S. Rommer, Phys. Rev. Lett. 75, 3537 (1995).

[14] D. Perez-Garcia et al., Quantum Inf. Comput. 7, 401 (2007), arxiv:quant-ph/0608197.

[15] F. Verstraete, D. Porras, and J.I. Cirac, Phys. Rec. Lett. 93, 227205 (2004). 
[16] H. Takasaki, T. Hikihara, and T. Nishino, J. Phys. Soc. Jpn. 68 , 1537 (1999); T. Nishino et al., Nucl. Phys. B575, 504 (2000).

[17] F. Verstraete and J. I. Cirac, arXiv:cond-mat/0407066.

[18] P.W. Anderson, Basic Notions of Condensed Matter Physics, Addison-Wesley: The Advanced Book Program, 1997, Reading, Mass.

[19] S. Coleman, An Introduction to Spontaneous Symmetry Breakdown and Gauge Fields, Laws of Hadronic Matter, Ed. A. Zichichi, Academic, 1975, New York.

[20] Although our examples are restricted to quantum systems on an infinite lattice in one spatial dimension, the argument is valid for quantum systems in two and higher dimensions. In the latter case, the TN algorithm based on PEPS [27] is necessary.

[21] E. Lieb, T. Schultz, and D. Mattis, Ann. Phys. 60, 407 (1961); P. Pfeuty, Ann. Phys. 57, 79 (1970).
[22] J.Kurmann et al., Physica A112, 235(1982); D. V. Dmitriev et al., J. Exp. Th. Phys. 95, 538 (2002); T. Roscilde, et al., Phys. Rev. Lett. 93, 167203 (2004).

[23] The extension of our argument to any other group is straightforward. It also applies to quantum lattice systems in any spatial dimensions.

[24] Moreover, one may establish the monotonicities of the reduced fidelity $F\left(\rho_{\lambda}, \rho_{\lambda^{\prime}}\right)$ with $\lambda$ for a fixed $\lambda^{\prime}$, along a similar line to the ground state fidelity per site [4], which may be used to identify stable fixed points.

[25] G. Vidal, Phys. Rev. Lett. 98, 070201 (2007).

[26] M. Suzuki, Phys. Lett. A146, 319 (1990).

[27] J. Jordan et al., arXiv:cond-mat/0703788 